\title{
An armed oncolytic adenovirus system, ZD55-gene, demonstrating potent antitumoral efficacy
}

\author{
Zi Lai ZHANG ${ }^{1 *}$, Wei Guo ZOU ${ }^{1 *}$, Chun Xia LUO ${ }^{1}$, Bing Hua LI ${ }^{1}$, Jin Hui WANG ${ }^{1}$, Lan Ying SUN ${ }^{1}$, Qi Jun \\ QIAN $^{2}$, XIN YUAN LIU ${ }^{1 * *}$ \\ ${ }^{1}$ Laboratory of Biotechnology, Institute of Biochemistry and Cell Biology, Shanghai Institutes for Biological \\ Sciences, Chinese Academy of Sciences, Shanghai 200031, China.E-mail:xyliu@sibs.ac.cn \\ ${ }^{2}$ Eastern Heptobiliary Hospital, Second Military Medical University, Shanghai 200438, China
}

\begin{abstract}
ONYX-015 is an attractive therapeutic adenovirus for cancer because it can selectively replicate in tumor cells and kill them. To date, clinical trials of this adenovirus have demonstrated marked safety but not potent enough when it was used alone. In this paper, we put forward a novel concept of Gene-ViroTherapy strategy and in this way, we constructed an armed therapeutic oncolytic adenovirus system, ZD55-gene, which is not only deleted of E1B 55-kD gene similar to ONYX-015, but also armed with foreign antitumor gene. ZD55-gene exhibited similar cytopathic effects and replication kinetics to that of ONYX-015 in vitro. Importantly, the carried gene is expressed and the expression level can increase with the replication of virus. Consequently, a significant antitumoral efficacy was observed when ZD55-CD/5-FU was used as an example in nude mice with subcutaneous human SW620 colon cancer. Our data demonstrated that ZD55-gene, which utilizing the Gene-ViroTherapy strategy, is more efficacious than each individual component in vivo.
\end{abstract}

Key words: ZD55-gene, oncolytic adenovirus, E1B 55-kD gene, cancer Gene-ViroTherapy.

\section{INTRODUCTION}

Human adenovirus vectors hold great promise as "drugs" to treat cancer[1]. For safety concern, most of adenoviruses used in clinical studies thus far carry a deletion in the E1 region, which renders the virus replication defective or helper-dependent. However, this defective also limited their use for cancer gene therapy as their inability to transduce and distribute throughout a tumor mass[2]. To address these problems, attentions have shifted to the use of conditionally replicative adenoviruses (CRAds) as the treatment platform.

CRAds represent a novel class of anticancer agents $[3,4]$, which are designed to selectively replicate in tumor cells and to destroy these cells by cytolysis. The release

\footnotetext{
*These two authors contributed equally to this work.

${ }^{* *}$ Corresponding author: Prof. Xin Yuan LIU, Tel: 86-21-64746126; Fax: 86-21-64746126; E-mail: xyliu@sibs.ac.cn

Abbreviations: ZD55, recombinant adenovirus with E1B 55-kD gene deletion; NHLF, normal human lung fibroblast; MOI, multiplicity of infection; PFU, plaque-forming unit(s); CPE, cytopathic effect; MTT, 3-(4,5-dimethylthiazol-2-yl)-2,5-diphenyltetrazolium bromide.

Received Oct-9-2003 Revised Oct-24-2003 Accepted Nov-12-2003
}

of viral progeny from lysed tumor cells offers the potential to amplify CRAds in situ and to achieve lateral spread to neighboring cells in a solid tumor, thus expanding the oncolytic effect. Moreover, the relative specificity of viral replication in tumor versus normal cells will dictate their safety and efficacy. Currently, CRAd-based cancer treatments are already being evaluated in clinical trials [5-7]. Among them, a mutant adenovirus known as ONYX-015 (originally termed $d l 1520$ ) is an elegant example, which was engineered not to express the E1B55-kD viral protein and was originally reported to preferentially target and destroy p53-dysfunctional tumor cells but not the adjacent normal tissue[8,9]. Now, it shows unambiguous evidence of antitumour activity in a broader range of tumors than initially anticipated[10-13].

Although these CRAds hold promises as anti-cancer agents, clinical experiences show that these agents alone are not potent enough to generate sustained clinical responses or to cause complete tumor regressions. In recent clinical trials, better results of ONYX-015 has been achieved when it was combined with some chemo- 
gherapeutic agents (5-FU, cisplatin) than used alone[14, 15]. However, other strategies need to be developed to increase its antitumor efficacy. One approach is to create less attenuated (more potent) adenoviruses. Reduced replication and cytopathogenicity have been reported for ONYX-015 when compared with wild-type Ad5[13], for E1B-55kD is known to facilitate the preferential transport of viral mRNA during the late stage of a lytic adenoviral infection [16]. Thus, less attenuating mechanisms such as making more precise mutations in the E1B gene can be employed. A report describes the identification of an E1B-55kD mutant, R240A, which fails to degrade p53 but has retained its E4orf6-binding and mRNA-transporting potential[17]. Another way is to combine this kind of adenovirus with therapeutic gene. Beyond the direct lytic function of virus, additional cytotoxic effects may be capable of eliminating tumor cells that the virus alone cannot achieve. For example, an E1B55K-deleted adenovirus has been devised, expressing herpes simplex virus-thymidine kinase(HSV-TK), which catalyzes phosphorylation of gancyclovir resulting in highly cytotoxic metabolites[2].

Given the potential and shortcoming of ONYX-015 as an antitumor agent, in this paper, we constructed an adenovirus named ZD55, in which E1B 55-kD gene was deleted similar to ONYX-015 but in a much easier way. Different from ONYX-015, which is an adenovirus hybrid of serotypes 2 and 5, ZD55 is derived only from Ad5. Moreover, ZD55 has an important character as it has been added with a clone site for easy insertion of foreign gene.

Furthermore, we considered the potential of combining therapeutic gene therapy with oncolytic virus therapy and put forward a novel concept of Gene-ViroTherapy strategy. In this way, we developed an armed oncolytic adenovirus system, ZD55-gene, in which the foreign therapeutic gene was inserted into ZD55. It was demonstrated that ZD55-gene carried all the advantages of ONYX-015, including specifically replication and induce cytopathic effects in tumor cells. In addition, ZD55-gene showed great improvement in its antitumor efficacy bcause of the additional function of therapeutic gene.

\section{MATERIALS AND METHODS}

\section{Cell lines and culture}

The following cell lines were used: human colorectal cancer cell line SW620, HCT116, human hepatocarcinoma cell line BEL7404, human cervical cancer cell line Hela, human normal lung fibroblasts cell line NHLF, human normal embryonic lung cell line MRC5, WISH, and human normal liver cell line L02 were purchased from ATCC (American Tissue Culture Collection, Rockville, MD); human breast cancer cell line Bcap37 was purchased from Shanghai Cell Collection, Chinese Academy of Sciences (CAS); HEK293 cell line (human embryonic kidney cells containing the E1A region of Adenovirus) was obtained from Microbix, Inc (Ontario, Canada).

SW620, BEL7404, Hela, MRC5, L02 and HEK293 cells were cultured in DMEM (GIBCO BRL, Grand Island, NY) supplemented with $10 \%$ heat-inactivated fetal bovine serum (FBS, GIBCO BRL), $4 \mathrm{~m} M$ glutamine, $50 \mathrm{U} / \mathrm{ml}$ penicillin and $50 \mu \mathrm{g} / \mathrm{ml}$ streptomycin. Bcap37, NHLF and WI-38 cells were grown in RPMI1640 supplemented with $10 \%$ FBS.

\section{Construction of pZD55}

pXC1 (Microbix Biosystems, Ontario, Canada) contains the Ad5 nucleotides 22-5790, including the inverted terminal repeat, the packaging sequences and the E1A and E1B genes inserted to pBR322 [20]. To delete the E1B55 region, nested PCR using pXC1 as the template were performed. The viral region comprising nucleotides 1321-2040 was amplified using a primer set of 5'- GCC GAC ATC ACC TGT G TCT AGA GAA TG -3' (Xba I primer, bp1321-1348) and 5'- TCA GAT GGG TTT CTT CAC TCC ATT TAT CCT-3' (Xba II primer, bp2040-2011). The region containing nucleotides 2007-2268 was amplified with another primer set of 5'-ATA AAG GAT AAA TGG AGT GAA GAA ACC CAT CTG AG-3' (Bgl I primer, bp2007-2041, the third codon of E1B 55-kD gene was mutated to premature translation stop codon with base $2024 \mathrm{C} \rightarrow \mathrm{T}$ ) and 5'-GA AGA TCT ATA CAG TTA AGC CAC CTA TAC AAC A-3' (Bgl II primer, bp2244-2268, two mutations at bases $2252 \mathrm{C} \rightarrow \mathrm{T}$ and $2261 \mathrm{G} \rightarrow \mathrm{T}$ generating premature translation stop codons in the 55$\mathrm{kD}$ E1B reading frame at codons 79 and 82 , respectively). Using the mixture of the two PCR fragments was as a template, a $955 \mathrm{bp}$ fragment was then amplified using $X b a$ I primer and $B g l$ II primer as above. This PCR product was cloned into $\mathrm{pXC1}$ to generate the plasmid pXC1-d55. SV40 polyA (160bp) were obtained through using pIRESpuro as temple and the following 5'-TGT GGA TCC TCT AGA GCT CGC TGA-3' (BamH I) and 5'-TCT AGA TCT CGA GCC CCA GCT GGT-3' ( $B g l$ II) as primers. Then it was cloned into $\mathrm{pXC1-d55}$ to generate the plasmid pZD55. The correct construction of this vector was confirmed by DNA sequencing. The entire expression cassette of foreign gene/genes was subcloned into pZD55 to generate the plasmid pZD55-gene.

\section{Generation of virus ZD55-gene}

A restricted replication-competent adenovirus ZD55-gene was constructed by standard homologous recombination techniques using the plasmid pZD55-gene and the adenovirus packaging plasmid pBHGE3 (adenovirus packaging plasmid, Microbix Biosystems, Ontario, Canada) in HEK293 cells. $1 \mu \mathrm{g}$ pZD55-gene was mixed with $0.5 \mu \mathrm{g}$ pBHGE3 and then co-transfected into HEK293 cells using Effectene Transfection Reagent (Qiagen, Germany). Recombinant adenovirus was isolated from a single plaque and expanded in HEK293 cells. A standard replication-deficient adenovirus Ad-gene was constructed using pCA13-gene and pBHGE3 as above. In this paper, ZD55-EGFP, ZD55-luc, ZD55-CD and Ad-CD were also constructed for use.

Other adenovirus Ad-EGFP, Ad- $\beta$ Gal (both are replication-defe- 
ctive Ad5 without E1 and E3 but containing the cytomegalovirus immediately early promoter driving the EGFP gene or $\beta$-gal gene) and ONYX-015 were from Dr. Qi Jun QIAN's lab.

Viruses were plaque purified, propagated on HEK293 cells and purified by $\mathrm{CsCl}$ gradient according to standard techniques. Particle titers of all adenoviruses were determined by absorbance measurements at $260 \mathrm{~nm}$, and functional PFU titers were determined by plaque assay on HEK293 cells.

\section{Western blot analysis of E1A, E1B 55-kD expression}

Cells were infected with ZD55-EGFP, Ad-EGFP and ONYX015 respectively at MOI of $10.48 \mathrm{~h}$ later, cells were washed with PBS and then lysed in SDS-PAGE sample buffer (62.5 $\mathrm{m} M$ Tris-Hcl, pH 6.8, 2\% SDS, $10 \mathrm{~m} M$ glycerol, 1.55\% DTT). Total cell lysates (30 $\mu \mathrm{g}$ of protein) were separated by $12 \%$ SDS-PAGE and then transferred to nitrocellulose membranes (Amersham). The level of E1A and E1B 55-kD protein was determined by Western blot analysis with the rabbit polyclonal antibody anti-Ad2 E1A (SC-403, Santa Cruz Biotech) and rabbit polyclonal antibody anti-Ad5 E1B 55-kD (D01492-3, Oncogene ${ }^{\mathrm{TM}}$ ) respectively. Reactivity was visualized by enhanced chemiluminescence (ECL) system (Amersham life Sciences Inc., Arlington Heights, Illinois).

\section{Cytopathic effect assay}

Five tumor cell lines (SW620, HCT116, BEL7404, Bcap37, Hela) and three normal cell lines (WISH, NHFL, MRC-5) were grown to sub-confluent and were uninfected or infected with ZD55-EGFP, ONYX-015, Ad-EGFP respectively at various MOI respectively. 7 days after infection, cells were exposed to $2 \%$ crystal violet in $20 \%$ methanol for $15 \mathrm{~min}$, then washed with distilled water and documented as photographs.

To quantify the cytopathic effect, cells were plated in triplicate to low density in 96-well plates and either mock-infected, or infected with ZD55-EGFP, ONYX-015, Ad-EGFP respectively at various MOI. Cell proliferation was assayed at the indicated time intervals by a method based on the metabolization of MTT (Sigma).

\section{Virus progeny production assay}

To determine virus progeny production, $2 \times 10^{5}$ cells were seeded in 6-well plates. $24 \mathrm{~h}$ later, cells were infected with $10^{5} \mathrm{PFU}$ of ZD55-EGFP or ONYX-015 or Ad-EGFP. After an additional $48 \mathrm{~h}$, medium and cells were collected, freeze-thaw for 3 cycles, and centrifuged to collect the supernatant. The production was determined by standard plaque assay on 293 cells.

\section{Reporter gene assay}

EGFP as reporter gene: cells were plated at a density of $10^{5}$ cells $16 \mathrm{~cm}$ dish and infected with ZD55-EGFP or Ad-EGFP at an MOI of 5. After $24 \mathrm{~h}$ and $120 \mathrm{~h}$, the expression of EGFP was assessed using Zeiss fluorescence microscope coupled with a digital Nikon camera photo apparatus.

To assess viral armed gene expression in vivo, s.c. grown xenografts of SW620 cells on nude mice were intratumorally injected with $5 \times 10^{8}$ viral particles of Ad-EGFP or ZD55-EGFP. 10 days later, tumors were harvested and were shock frozen in liquid nitrogen. Thin slices of native tumor samples were screened for EGFP expression under a fluorescence microscope.

Luciferase and $\beta$-galactosidase as reporter genes: cells were plated at a density of $3 \times 10^{4}$ cells/well and infected with ZD55-Luc or Ad$\beta \mathrm{Gal}$ at a MOI of 0.01 . After infection, cells were harvested every 24 $\mathrm{h}$ for six days and lysed in the reporter lysis buffer (Promega, Madison, WI). The luciferase activity was detected using Luciferase Assay System Freezer Pack Kit (Promega Madison, WI) in a microtiter plate luminometer (BG-P) and the chemiluminescent $\beta$ galactosidase assay was performed using Luminescent $\beta$-galactosidase Reporter Systerm3 kit (BD Biosciences Clontech).

\section{Animal tumor model studies}

All animal experients were carried out in accordance with the National Institute of Health Guide for the Care and Use of Laboratory Aniamls.

Female Balb/c nude mice at 4-5 weeks were obtained from the Animal Research Committee of the Institute of Biochemistry and Cell Biology (Shanghai, China) and quarantined for a week before tumor implantation. SW620 human colon tumor xenografts model was established by subcutaneously injecting $2 \times 10^{6}$ SW620 cells into the right flank of mice. Tumor growth was measured weekly and tumor volume $(\mathrm{V})$ was calculated as the formula: $\mathrm{V}\left(\mathrm{mm}^{3}\right)=$ lengthxwidth ${ }^{2} / 2$. Once the tumors reached a volume of $100-150 \mathrm{~mm}^{3}$, animals were randomly divided into ZD55-CD/5-FC, Ad-CD/5-FC, ONYX-015 and PBS groups. A daily dose of $5 \times 10^{8} \mathrm{PFU}$ suspended in $100 \mu \mathrm{l}$ of PBS or $100 \mu \mathrm{l}$ PBS alone was administrated intratumorally for 3 days. After virus inoculation, 5-FC (Sigma) $30 \mathrm{mg} / \mathrm{kg}$ of body weight was administered i.p. in $1 \mathrm{ml}$ of PBS for 5 consecutive days. Survival animals were sacrificed 80 days after tumor cells injection.

\section{Statistical analysis}

All data were displayed as Mean \pm S.D. and were processed by the statistical software SPSS 10.1. Statistical significance was assumed when $\mathrm{p} \leq 0.05$.

\section{RESULTS}

\section{Construction of pZD55-gene and ZD55-gene}

pZD55 were constructed as described in the materials and methods. Restrictive enzyme digestion assay and sequence assay (data not shown) both confirmed the correct construction of this vector. As shown in Fig 1A, using $X b a$ I and $B g l$ II, 1989bp, 931bp and 1090bp can be cut from $\mathrm{pXC1}$, pXC1-d55 and pZD55 respectively, which suggests that pZD55 deletes E1B 55-kD gene and SV40 polyA is inserted in the right direction after E1B19-kD gene. pZD55-gene can be constructed by inserting the whole foreign gene expression cassette cutted from pCA13-gene using $B g l$ II into the corresponding site of pZD55 directly.

A schematic structure of ZD55-gene, which was designed to express the wide-type adenovirus $5 \mathrm{E} 1 \mathrm{~A}$ and $19-\mathrm{kD}$ E1B proteins is shown in Fig 1B. Three premature translation stop codons were placed in the 55-kD E1B 
gene that would result in the production of a truncated 78-amino acid protein, which does not contain the p53 binding domain (amino acids 215-354) and therefore cannot interact with $\mathrm{p} 53$. Expression of the foreign gene is driven by the human CMV promoter (or other specific promoters/controlled promoters) and utilizes SV40 polyA element. In this adenovirus system, E3 region is reserved, which was reported to enhance the viral spread and oncolysis[18].

To demonstrate that ZD55-gene had the expected properties, virus-infected SW620 colon cancer cells were examined for the expression of the E1A, E1B 55$\mathrm{kD}$ proteins (ZD55-EGFP was used as an example of ZD55-gene in this paper). ZD55-EGFP produced similar amount of the E1A protein as wide-type Ad5 and ONYX015. However, like ONYX-015, ZD55-EGFP failed to express the E1B 55-kD protein (Fig 1C).

\section{Tumor selective cytopathic effect of ZD55-EGFP}

To determine whether ZD55-gene keep the viral characters as that of ONYX-015, an in vitro CPE assay was preformed to evaluate the cytopathic effect of ZD55-gene. A set of tumor cell lines (Bcap37, BEL7404, HCT116,
Hela) and normal cell lines (WISH, MRC5) were infected with ZD55-EGFP at various MOIs. ONYX-015 and AdEGFP were infected in parallel as control. As shown in Fig 2A, ZD55-EGFP exerted marked CPE against all tumor cell lines tested. All these cells were sensitive to ZD55-EGFP at low MOI of 1. Its cytopathic capacity was comparable to that of ONYX-015 and was about 100 times greater than that of Ad-EGFP. When tested in normal cells, all these three viruses caused no significant CPE even when concentrations were up to $100 \mathrm{PFU}$ per cell. In special, both ZD55-EGFP and ONYX-015 caused about 100 -fold attenuation in killing normal cells than killing tumor cells, suggesting that ZD55-EGFP can induce tumor-specific cytopathic effect as ONYX-015.

To further quantify the cytopathic effect of ZD55gene, cell viability was examined. As shown in Fig 2B, at all titers, the number of surviving cells after infecting with ZD55-EGFP was nearly similar to ONYX-015 in SW620 cells. Their cytopathic effect was dosage and time-dependent. However, no significant differences were observed when comparing the cytopathic effect of ZD55-EGFP, ONYX-015 and Ad-EGFP in NHFL cells. Similar results were obtained from other tumor cell
A
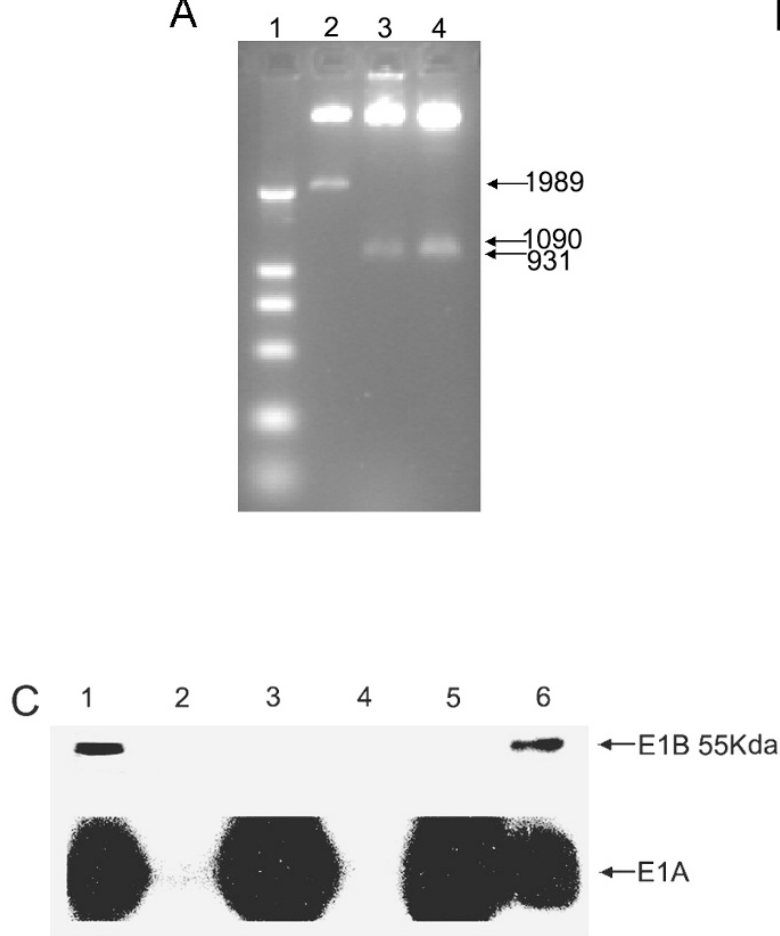

B

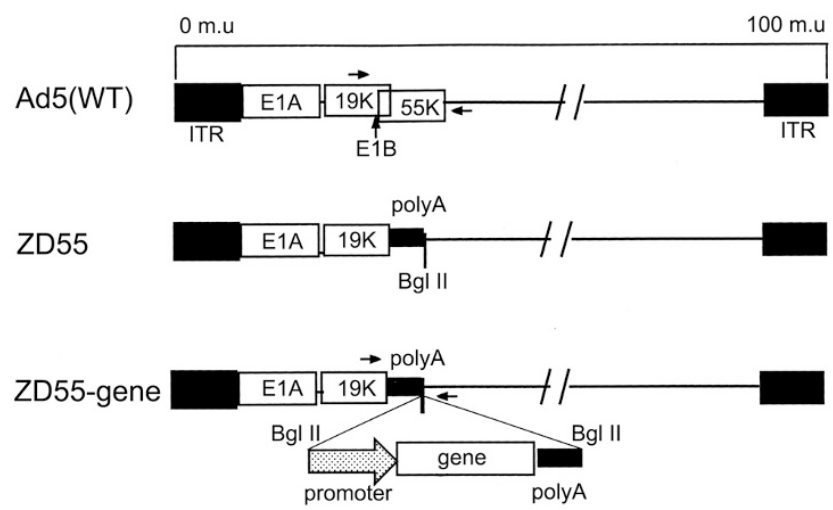

Fig 1. Construction and Characterize of ZD55-gene. (A) Identification of pZD55 plasmid. 1. L2000 Marker; 2. PXC1/ XbaI +BglII; 3.pXC1D55/ XbaI +BglII; 4. pZD55/ XbaI +BglII.(B) Schematic structure of ZD55-gene. In ZD55-gene, the E1B 55-kD gene (bp2269-3327) was replaced by SV40 polyA and the expression box of foreign gene. (C) Western blot assay of E1A and E1B 55-kD gene expression by ZD55-EGFP.SW620 cells were mock-infected (lane 2) or infected with ONYX-015 (lane 3) Ad-EGFP (E1'E3- replication defective vector, lane 4), ZD55-EGFP (lane 5) and Ad-WT (wild-type adenovirus, lane 6). Lysates from 293 cells were included as a positive control (lane 1). Lysates from uninfected and infected cells were subjected to Western blot assay with anti-Ad2 E1A Ab and anti-Ad2 E1B 55-kD Ab. 

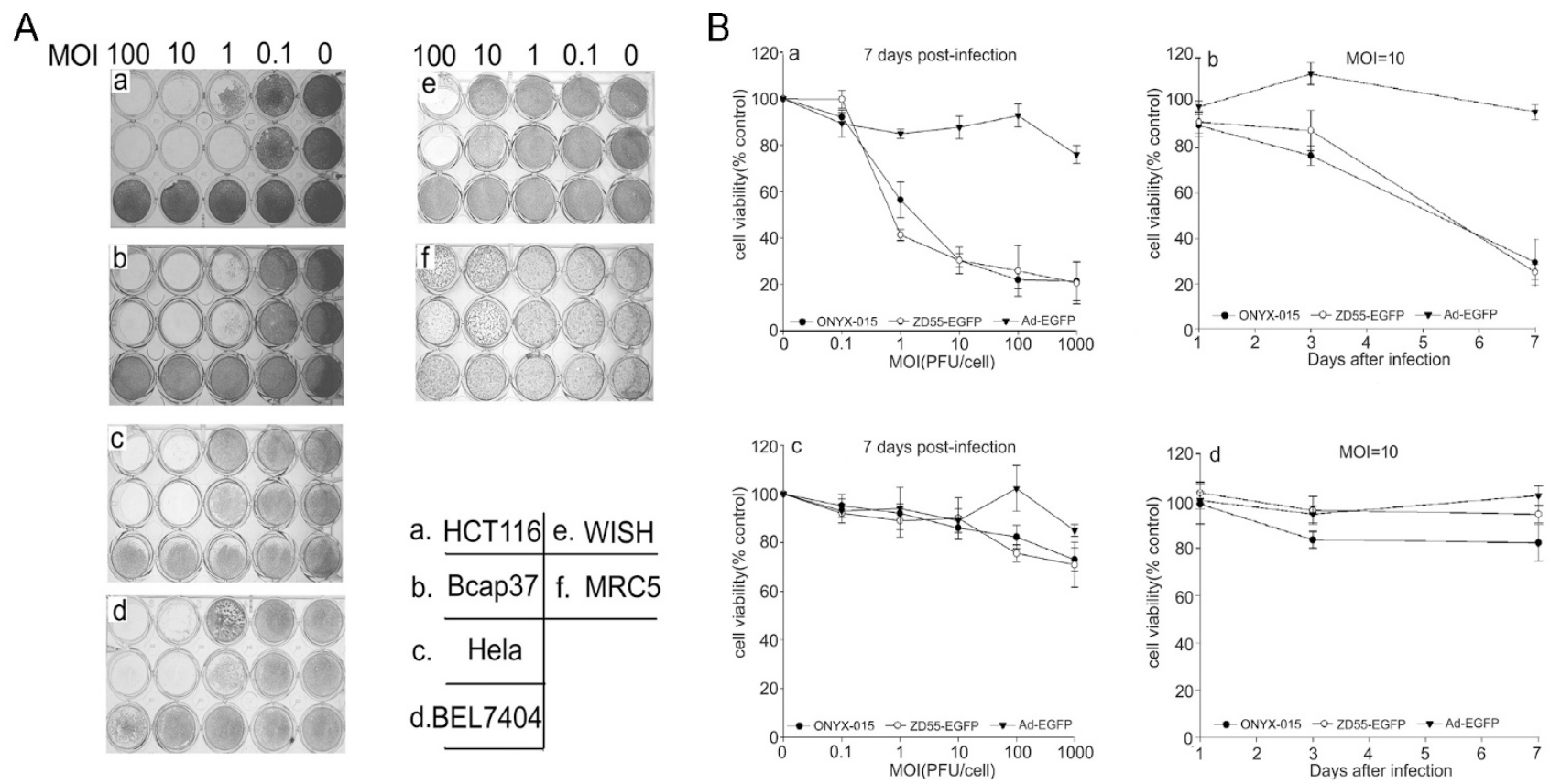

Fig 2. Tumor-selective cytopathic effect of ZD55-EGFP (A) Tumor cells (Hela, Bcap37, BEL7404, HCT116) and normal cells (MRC5, WISH) were infected with ZD55-EGFP, ONYX-015 and Ad-EGFP at indicated MOIs. 7 days later, cells were stained with crystal violet. (B) SW620 cells (a and $\mathbf{b})$ and NHLF cells (c and $\mathbf{d})$ were infected with increasing MOIs or indicated MOI (MOI=10) of Zd55-EGFP, ONYX015 and Ad-EGFP. After 1, 3 and 7 days post-infection, cells were subjected to MTT assay. Results are Mean \pm SD (bars) of triplicates and

lines and normal cell lines (data not shown).

\section{Tumor selective replication of ZD55-EGFP}

According to the mechanism of oncolytic virus, the most important character is its ability of selective replicating in tumor cells[3]. To compare the replication ability between ZD55-gene and that of ONYX-015, tumor cell lines (SW620, Bcap37, BEL7404, Hela) and normal cell lines (NHLF and ECV304) were infected with $10^{5} \mathrm{PFU}$ of ZD55-EGFP or ONYX-015, the accumulation of viral particles in culture medium and cell fraction was determined by plaque assay on 293 cells. As shown in Fig 3, total viral yields of ZD55-EGFP and ONYX-015 were similar, both showed much stronger specific replication capability, producing 100 to 2000 -fold more virus in tumor cells than in normal cells. All these results demonstrate that ZD55-gene can selectively replicate in tumor cells and the replication properties correlate remarkably with its cytopathic effect.

Increased expression of foreign gene by ZD55-gene

Compared with ONYX-015, the most distinguishing character of ZD55-gene is that foreign gene can be easily inserted into and its expression level can be amplified with the replication of adenovirus. As shown in Fig 4A, although both ZD55-EGFP and Ad-EGFP showed very

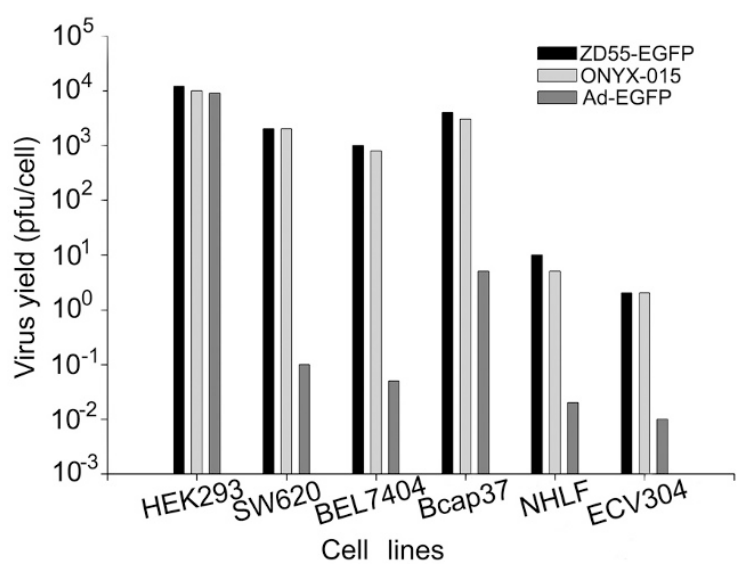

Fig 3. Tumor-selective replication of ZD55-EGFP. Tumor cells (Hela, Bcap37, BEL7404, SW620) and normal cells (NHLF, ECV304) were infected with $10^{5}$ PFU of ZD55-EGFP, ONYX-015 and AdEGFP, respectively. After an additional $72 \mathrm{~h}$, medium and cells were scraped and subjected to three freeze-thaw cycles. The collected supernatant was tested for virus production by standard plaque assay on 293 cells. The data shown are Mean of two independent experiments. 
A
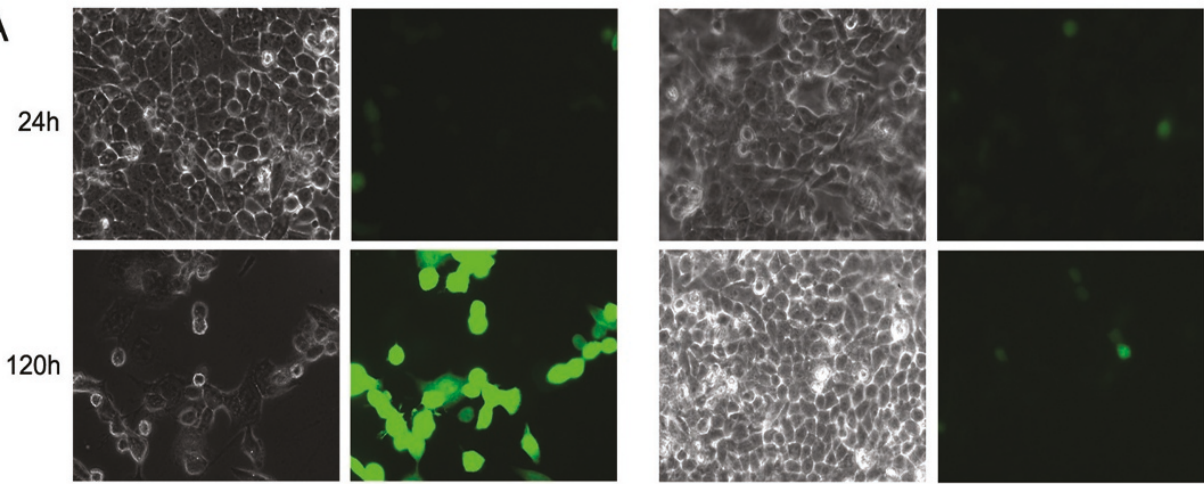

ZD55-EGFP

Ad-EGFP
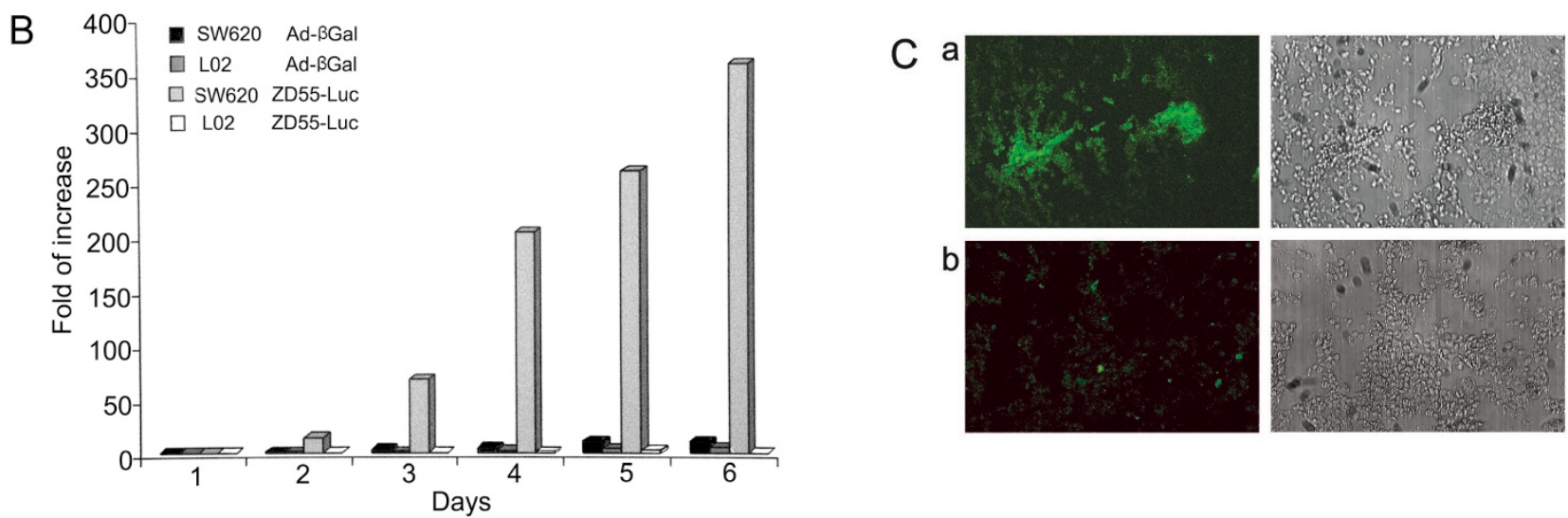

b
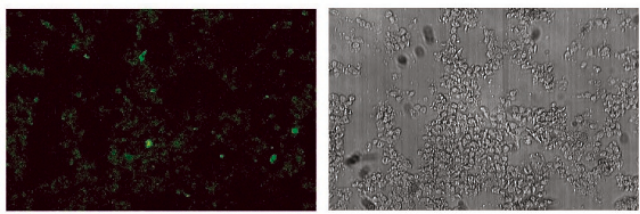

Fig 4. Increased expression of foreign gene by ZD55-gene. (A) EGFP as a reporter gene. BEL7404 cells were infected with ZD55-EGFP and Ad-EGFP at an MOI of 2. EGFP expression was assessed by photomicrographic examination at $24 \mathrm{~h}$ and $120 \mathrm{~h}$ after infection. (B) Luciferase as a reporter gene. SW620 cells and L-02 cells were infected with Ad- $\beta$ Gal and ZD55-Luc at an MOI of 0.01.The expression of $\beta$-gal and luciferase was calculated as the fold of the expression of the first day. The data shown are Mean of two independent experiments. (C) ZD55gene can highly express its armed gene in vivo. SW620-dervied s.c. tumor xenografts on nude mice were intratumorally injected with $5 \times 10^{8}$ viral particles of ZD55-EGFP (a) and Ad-EGFP (b), respectively. 10 days later, effective viral spreading could be observed using EGFP fluorescence in tumor samples.

low EGFP expression after 1 day infection in BEL7404 cells, the former showed much higher EGFP expression than the later after 5 days. Moreover, significant cytotoxicity was observed in ZD55-EGFP infected BEL7404 cells while not in Ad-EGFP infected BEL7404 cells. The expression level of EGFP was especially high in these undergoing cytopathic cells. In MRC-5 cells, no cytotoxicity was observed both in ZD55-EGFP and Ad-EGFP and the EGFP expression were both restricted in few cells (data not shown).

ZD55-Luc was used as another system to quantitatively measure foreign gene expression of ZD55-gene in tumor cells. Cells were infected with ZD55-Luc at very low MOI of 0.01 . In SW620 cells, luciferase activity induced by ZD55-Luc increased significantly, which was as high as 356-fold when compared that of day 6 to day 1. However, in L-02 cells, luciferase inducement was very low. Meanwhile, Ad- $\beta$ Gal was used as a control to infect both SW620 cells and L-02 cells, as it lost its replication competence, its $\beta$-gal expression level increased slightly with time in both cell lines tested (Fig 4B).

In addition, to assess whether the armed gene of ZD55-gene can be highly expressed in vivo, SW620dervied s.c. tumor xenografts on nude mice were intratumoral injected with a single dose of ZD55-EGFP and Ad-EGFP, respectively. Monitoring EGFP expression in native tumor samples revealed that ZD55-EGFP could effectively spread through the tumor tissue meanwhile express much higher level of its armed gene than that of Ad-EGFP (Fig 4C).

\section{Antitumoral efficacy of ZD55-CD in nude mice}

Encouraged by the effect of ZD55-gene in vitro, we sought to evaluate its antitumor efficacy in vivo. Prodrug-based therapies, such as using Escherichia coli $\mathrm{CD} / 5-\mathrm{FC}[19]$ system, have been administered using 
a variety of vectors into various cancers. However, these therapies have not generated meaningful benefit in the clinical trails, presumably due to the poor distribution of delivery vehicles[20]. To our knowledge, oncolytic adenovirus has not been reported as the vector to carry $\mathrm{CD}$ gene, so we chose ZD55-CD/5-FC for our in vivo studies to illustrate the efficacy of ZD55-gene.

A SW620 human colon cancer xenograft model was established in nude mice. Once the tumor's volume

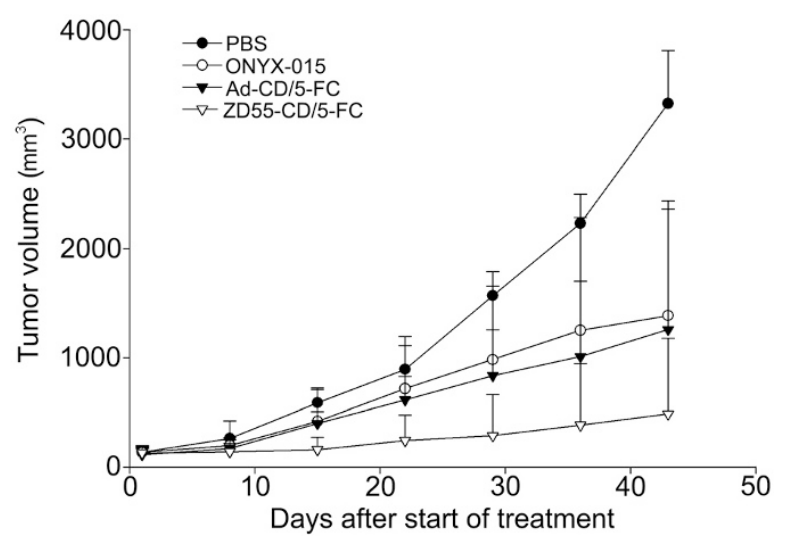

Fig 5. ZD55-CD in combination with 5-FC has potent antitumoral efficacy on SW620 xenograft tumors. Tumor xenografts were established by s.c. injecting $2 \times 10^{6}$ SW620 cells suspended in $100 \mu 1$ of serum free DMEM into the right flank of nude mice. When tumor volumes reached about $150 \mathrm{~mm}^{3}$, mice were randomized and intratumorally injected with $5 \times 10^{8} \mathrm{PFU}$ of ZD55-CD/5-FC $(\mathrm{n}=6)$, Ad-CD/5-FC $(n=6)$, ONYX-015 $(n=6)$, and PBS diluents $(n=6)$ daily for 3 days, then 5 -FC ( $30 \mathrm{mg} / \mathrm{kg}$ of body weight) was administered $\mathrm{i}$. p. in $1 \mathrm{ml}$ of PBS for 5 consecutive days. The tumor volumes were estimated as: Tumor volume $\left(\mathrm{mm}^{3}\right)=$ lengthxwidth ${ }^{2} / 2$.

reached about $100 \mathrm{~mm}^{3}$, animals were randomly assigned to treatment groups. To assess the antitumor effect of ZD55-CD in combination with 5-FC, a standard replication-defective Ad-CD in combination with 5-FC, ONYX015 and PBS were used as controls. Tumor growth curves were adopted to monitor the difference in treatment efficacy (Fig 5). On day 42, one of the six mice in PBS-treated and ONYX-015-treated group died and at that time, the mean tumor volumes of PBS, Ad-CD/5FC, ONYX-015 and ZD55-CD/5-FC-treated mice were $3324.349,1257.57,1385.948$ and $487.4732 \mathrm{~mm}^{3}$, respectively. One-way ANOVA (Tukey-Kramer test) demonstrated that although no significant difference was observed between ONYX-015 and Ad-CD/5-FC, the efficacy of ZD55-CD/5-FC was much higher than both Ad-CD/5-FC and ONYX-015 ( $p<0.05)$. On day 80, the end point of the study, two of six mice that received
ZD55-CD/5-FC were tumor free. While in all other treatment groups some animals had to be euthanized because their tumor size exceeded $10 \%$ of their body weight.

\section{DICUSSION}

The clear understanding of the complexity and plasticity of human solid tumors dictate that combination therapies will need to be employed to generate effective, durable responses for the cancer patient[21]. The clinical results of using ONYX-015 as a therapeutic agent validated this point that a significant antitumor activity was only seen when viral treatment was combined with chemotherapy $[14,15]$. In order to improve the efficacy of ONYX015 while avoiding the toxicity caused by systemic using chemotherapeutic agents, we presumed that the combination of ONYX-015 with therapeutic gene into a single vector might be a good choice. So, in this paper, we put forward a novel concept of Gene-ViroTherapy strategy and constructed an armed oncolytic adenovirus system named ZD55-gene, which is not only deleted of E1B 55-kD gene similar to ONYX-015, but also armed with foreign gene.

As ZD55-gene system takes advantage of the viruses' ability to selectively replicate and spread in the tumor mass to safely and efficiently deliver therapeutic genes to target tissues, it will effectively attack the complexity associated with human tumors[22]. The efficacy of this system was evaluated both in vitro and in vivo. Firstly, it was demonstrated that ZD55-gene system could keep its typical virus character similar to that of ONYX-015. Here, ZD55-EGFP was used as an example, which can specifically replicated and caused significant cytopathic effect in all tumor cells tested, while showed very low effect in normal cells tested. Then, the ability of ZD55gene system to high express its carried gene was validated through comparing its carried gene expression level with that of replication-deficient adenovirus. The much higher EGFP expression from ZD55-EGFP than that of AdEGFP was found and this result was also confirmed quantificationally by using ZD55-luc. Finally and most importantly, the superior antitumor efficacy to that of either ONYX-015 or replication-deficient adenovirus with therapeutic gene was demonstrated when we used ZD55$\mathrm{CD}$ in combination with 5-FC as an example. ZD55$\mathrm{CD} / 5$-FC treated mice exhibited much slow tumor growth rate than both Ad-CD/5-FC and ONYX-015 treated groups. More excitingly, at the end of this study, two of six mice that received ZD55-CD/5-FC were tumor free. 
Although ZD55-gene system exhibited its potential efficacy in our study, as a starting point, there are many problems need to be considered. For example, choosing the appropriate gene(s) with which to arm our ZD55 as ZD55-gene to enable it to arrest or eradicate tumor is a major question that has no simple answers. Several classes of gene therapy-based therapeutics have been traditionally associated with nonreplicating viral-based gene delivery vehicles (antioncogenes, tumor suppressor genes, prodrug-converting enzymes genes, antiangiogenic and immunology-based gene therapies). All of these therapeutic genes can be candidates for our use, and in our lab we have armed many therapeutic genes with ZD55 to construct a serial of virus, such as ZD55-CD, ZD55-Trail, ZD55-Smac, ZD55-Angiostatin, ZD55-sflt-1, ZD55-IL-12, ZD55-IL-24 and so on. The mechanisms of each of the various classes of gene based therapeutics when used as monotherapies may be clear, but their potential interactions within the context of a replicating adenovirus are not easily discerned[21]. In this paper, we armed prodrug gene-converting enzyme $\mathrm{CD}$ with ZD55, which generates the toxic chemotherapeutic drug 5-FU from the nontoxic prodrug 5-FC. As 5-FU may have potential antiviral activities associated with them, induction the expression of cytotoxic genes in later stages of infection would be most effective. Papers from Hermiston and colleagues have dealt with this issue by inserting toxin genes in the $\mathrm{E} 3$ region in place of the native adenoviral genes. They found that the replacement of E3-6.7K/gp19K, ADP, and E3B encoding regions with toxin genes resulted in a timing of expression similar to that of the replaced viral genes, thereby maintaining normal expression of the resident adenoviral genes [23, 24].

In addition, ONYX-015 was originally developed to replicate in and preferentially lyses p53-dysfunctional cells. However, recently unambiguous evidence showed that its antitumour activity is not completely dependent on p53[10-13]. As ZD55 deleted E155-kD gene similar to ONYX-015, this similar problem should be considered in our ZD55-gene system. So, the biological mechanisms defining the interaction of this virus with its human host need further exploration. Besides deleting E1B 55-kD gene to achieve tumor-selective adenovirus replication, other strategies were developed for this goal. For example, using tumor/ tissue-specific promoters (TSPs) to direct the expression of genes essential for viral replication, leading to the transcriptional targeting of viruses to cancer cells, has been widely exploited[2530]. A restricted-replication adenovirus using telomerase reverse transcriptase (hTERT) promoters to drive the expression of E1A was developed in our lab[31]. Another approach to achieve the specificity of adenoviruses is to modify the viral coat, for example, altering its receptor (CAR) specificity by utilizing other cancer specific proteins as alternate receptors[32-34]. In this way, it also can circumvent the problem of losing expression of adenovirus receptors on target cells.

Moreover, it is important to note that treatment with ZD55-gene does not exclude the use of chemotherapy, radiation, or surgery. On the contrary, we can presume that much better therapeutic effect can be achieved when these agents are used in combination with the viral-based therapy. For example, because previous reports indicated that the binding of adenovirus E1A proteins to the cellular retinoblastoma and p300 proteins can induce quiescent cells to enter the $\mathrm{S}$ phase[35], it is feasible that the combination of virotherapy and chemotherapy will further improved the treatment efficacy.

In conclusion, we constructed an armed oncolytic adenovirus system named ZD55-gene, which is a natural evolution of either the oncolytic virus-based therapy or the therapeutic gene-based therapy as it couple the lytic capability of adenovirus with the capacity to deliver therapeutic factors. Consequently, it provides an opportunity to generate a single agent that can attack tumors at multiple levels and represents a potentially exciting new treatment paradigm for human cancers.

\section{ACKNOWLEDGEMENTS}

This study was supported by the Key Project of the Chinese Academy of Sciences (No. KSCX2-3-06), the National Natural Science Foundation of China (No. 30120160823), and the Chinese National "863" High Tech Project Foundation grant (No. 2002AA216021). We would like to thank Ming ZHUO for professional technical assistance and for useful suggestions.

\section{REFERENCES}

1 Toth K, Doronin K, Tollefson AE, Wold WS. A multitasking oncolytic adenovirus vector. Mol Ther 2003; 7:435-7.

2 Wildner O, Morris JC. The role of the E1B $55 \mathrm{kDa}$ gene product in oncolytic adenoviral vectors expressing herpes simplex virus$t k$ : assessment of antitumor efficacy and toxicity. Cancer Res 2000; 60:4167-74.

3 Alemany R, Balague C, Curiel DT. Replicative adenoviruses for cancer therapy. Nat Biotechnol 2000; 18:723-7. 
4 Heise C, Kirn DH. Replication-selective adenoviruses as oncolytic agents. J Clin Invest 2000;105:847-51.

5 Khuri FR, Nemunaitis J, Ganly I, et al. A controlled trial of intratumoral ONYX-015, a selectively-replicating adenovirus, in combination with cisplatin and 5-fluorouracil in patients with recurrent head and neck cancer. Nat Med 2000; 6:879-85.

6 Nemunaitis J, Ganly I, Khuri F, et al. Selective replication and oncolysis in p53 mutant tumors with ONYX-015, an E1B-55kD gene-deleted adenovirus, in patients with advanced head and neck cancer: a phase II trial. Cancer Res 2000; 60:6359-66.

7 DeWeese TL, van der Poel H, Li S, et al. A phase I trial of CV706, a replication-competent, PSA selective oncolytic adenovirus, for the treatment of locally recurrent prostate cancer following radiation therapy. Cancer Res 2001; 61:7464-72.

8 Bischoff JR, Kirn DH, Williams A, et al. An adenovirus mutant that replicates selectively in p53-deficient human tumor cells. Science 1996; 274:373-6.

9 Heise CC, Williams AM, Xue S, Propst M, Kirn DH. Intravenous administration of ONYX-015, a selectively replicating adenovirus, induces antitumoral efficacy. Cancer Res 1999; 59 (11):2623-8.

10 Goodrum FD, Ornelles DA. The early region 1B 55-kilodalton oncoprotein of adenovirus relieves growth restrictions imposed on viral replication by the cell cycle. J Virol 1997; 71:548-61.

11 Rothmann T, Hengstermann A, Whitaker NJ, Scheffner M, zur Hausen H. Replicaton of ONYX-015, a potential anticancer adenovirus, is independent of p53 status in tumor cells. J Virol 1998; 72:9470-8.

12 Steegenga WT, Riteco N, Bos JL. Infectivity and expression of the early adenovirus proteins are important regulators of widetype and $\triangle \mathrm{E} 1 \mathrm{~B}$ adenovirus replication in human cells. Oncogene 1999; 18:5032-43.

13 Harada JN, Berk AJ. P53-independent and -dependent requirements for E1B-55K in adenovirus type 5 replication. J Virol 1999; 73:5333-44.

14 Khuri FR, Nemunaitis J, Ganly I, et al. A controlled trial of intratumoral ONYX-015, a selectively-replicating adenovirus, in combination with cisplatin and 5-fluorouracil in patients with recurrent head and neck cancer. Nat Med 2000; 6:879-85.

15 Heise C, Sampson-Johannes A, Williams A, McCormick F, Von Hoff DD, Kirn DH. ONYX-015, an E1B gene-attenuated adenovirus, causes tumor-specific cytolysis and antitumoral efficacy that can be augmented by standard chemotherapeutic agents. Nat Med 1997; 3:639-45.

16 Kratzer F, Rosorius O, Heger P, et al. The adenovirus type 5 E1B-55K oncoprotein is a highly active shuttle protein and shuttling is independent of E4orf6, p53 and Mdm2. Oncogene 2000; 19:850-7.

17 Shen Y, Kitzes G, Nye JA, Fattaey A, Hermiston T. Analyses of single-amino-acid substitution mutants of adenovirus type 5 E1B55K protein. J Virol 2001; 75:4297-307.

18 Yu DC, Chen Y, Seng M, Dilley J, Henderson DR. The addition of adenovirus type 5 region E3 enables calydon virus 787 to eliminate distant prostate tumor xenografts. Cancer Res 1999; 59:4200-3.
19 Kievit E, Nyati MK, Ng E, et al. Yeast Cytosine Deaminase Improves Radiosensitization and Bystander Effect by 5Fluorocytosine of Human Colorectal Cancer Xenografts. Cancer Res 2000; 60:6649-55.

20 van Dillen IJ, Mulder NH, Vaalburg W, de Vries EF, Hospers GA. Influence of the bystander effect on HSV- tk/GCV gene therapy. A review. Curr Gene Ther 2002; 2:307-22.

21 Hermiston TW, Kuhn I. Armed therapeutic viruses: Strategies and challenges to arming oncolytic viruses with therapeutic genes. Cancer Gene Ther 2002; 9:1022-35.

22 Hermiston T. Fighting fire with fire: attacking the complexity of human tumors with armed therapeutic viruses. Curr Opin Mol Ther 2002; 4:334-42.

23 Hawkins LK, Hermiston TW. Gene delivery from the E3 region of replicating human adenovirus: Evaluation of the ADP region. Gene Ther 2001; 8:1132-41.

24 Hawkins LK, Johnson L, Bauzon M, et al. Gene delivery from the E3 region of replicating human adenovirus: Evaluation of the $6.7 \mathrm{~K} / \mathrm{gp} 19 \mathrm{~K}$ region. Gene Ther 2001; 8:1123-31.

25 Gomez-Navarro J, Curiel DT. Conditionally replicative adenoviral vectors for cancer gene therapy. Lancet Oncol 2000; 1:14858.

26 Kirn D, Martuza RL, Zwiebel J. Replication selective virotherapy for cancer: Biological principles, risk management and future directions. Nat Med 2001; 7:781-7.

27 Rodriguez R, Schuur ER, Lim HY, Henderson GA, Simons JW, Henderson DR. Prostate attenuated replication competent adenovirus (ARCA) CN706: A selective cytotoxic for prostate-specific antigen-positive prostate cancer cells. Cancer Res 1997; 57: 2559-63

28 Hallenbeck PL, Chang YN, Hay C, et al. A novel tumor-specific replication-restricted adenoviral vector for gene therapy of hepatocellular carcinoma. Hum Gene Ther 1999; 10:1721-33.

29 Brunori M, Malerba M, Kashiwazaki H, Iggo R. Replicating adenoviruses that target tumors with constitutive activation of the wnt signaling pathway. J Virol 2001; 75:2857-65.

30 Kurihara T, Brough DE, Kovesdi I. Kufe DW. Selectivity of a replication-competent adenovirus for human breast carcinoma cells expressing the MUC1 antigen. J Clin Invest 2000; 106:76371.

31 Zou WG, Luo CX, Zhang ZL, et al. A novel hTERT promoterdriven oncolytic adenovirus demonstrates potent and selective antitumoral efficacy. Oncogene 2003; 23:1-8.

32 Suzuki K, Fueyo J, Krasnykh V, Reynolds PN, Curiel DT, Alemany R. A conditionally replicative adenovirus with enhanced infectivity shows improved oncolytic potency. Clin Cancer Res 2001; 7:120-6.

33 Shinoura N, Yoshida Y, Tsunoda R, et al. Highly augmented cytopathic effect of a fiber-mutant E1B-defective adenovirus for gene therapy of gliomas. Cancer Res 1999; 59:3411-6.

34 Wickham TJ. Targeting adenovirus. Gene Therapy 2000; 7:1104.

35 Sanchez-Prieto R, Quintanilla M, Cano A, et al. Carcinoma cell lines become sensitive to DNA-damaging agents by the expression of the adenovirus E1A gene. Oncogene 1996; 13:1083-92. 